\section{A child with Crohn disease}

\section{Chandra Abeysekera ${ }^{1}$, Navam Kumarasinghe ${ }^{2}$}

Sri Lanka Journal of Child Health, 2007; 36: 27-30

(Key words: Crohn disease, children)

\section{Introduction}

Crohn disease is an idiopathic, chronic inflammatory disorder of the bowel, involving any region of the gastrointestinal tract from the mouth to anus. It is commonly seen in North America and Northern Europe but is uncommon in Sri Lanka. This is a report of a Sri Lankan child with Crohn disease.

\section{Case report}

Ten year old Anushika from Ginigathhena presented with abdominal pain of 3 months duration. This pain was episodic involving the right side of the abdomen and the frequency of episodes and the severity of pain increased with time disturbing her routine activities. During this period she had lost 2 kilograms in weight. There was no alteration of her bowel habits. She had no other symptoms. She failed to respond to treatment taken from several local hospitals and was admitted for further evaluation.

She is the second of three children. She was born at term to healthy non-consanguineous parents following an uneventful pregnancy. Her birth weight was $3.2 \mathrm{~kg}$. Her growth was retarded from the beginning for which her parents were reassured. She had no significant illnesses in the past and her mental development and immunizations were up to date. There were no significant illnesses in her family. There was no contact history of tuberculosis (TB).

On examination, her growth parameters were well below the 3rd percentile for her age. She was pale and was in discomfort. The only positive finding on system examination was an ill-defined, intra abdominal mass in the right hypochondrium with mild tenderness.

Her haemoglobin $(\mathrm{Hb})$ was $7.7 \mathrm{~g} / \mathrm{dl}$ and the other

${ }^{1}$ Head \& Senior Lecturer, ${ }^{2}$ Assistant Lecturer, Department of Paediatrics, Faculty of Medicine. University of Peradeniya

(Received on 28 April 2006. Accepted on 22 June 2006) haematological indices, including the blood picture, indicated a hypochromic, microcytic anaemia. White cells count (WBC) showed absolute neutrophil leucocytosis and platelet count was $936 \times 10^{9} / \mathrm{L}$. Her ESR was $93 \mathrm{~mm} / 1 \mathrm{st}$ hour, total proteins $71.5 \mathrm{~g} / \mathrm{l}$ and albumin $28.9 \mathrm{~g} / 1$. All other laboratory investigations, including serum amylase, renal functions, liver functions, random blood sugar, urine and stool tests and chest $\mathrm{x}$ ray, were normal.

Ultrasound scan of abdomen showed a thickened transverse colon $(7 \mathrm{~mm})$ including hepatic flexure and a thickened mesentery. Other organs were normal. This appearance was suggestive of intestinal TB or a lymphoma.

Colonoscopy (figure 1) showed discrete areas of punctuate, linear and snail tract ulcers in the mucosa with inflammation suggestive of Crohn disease.

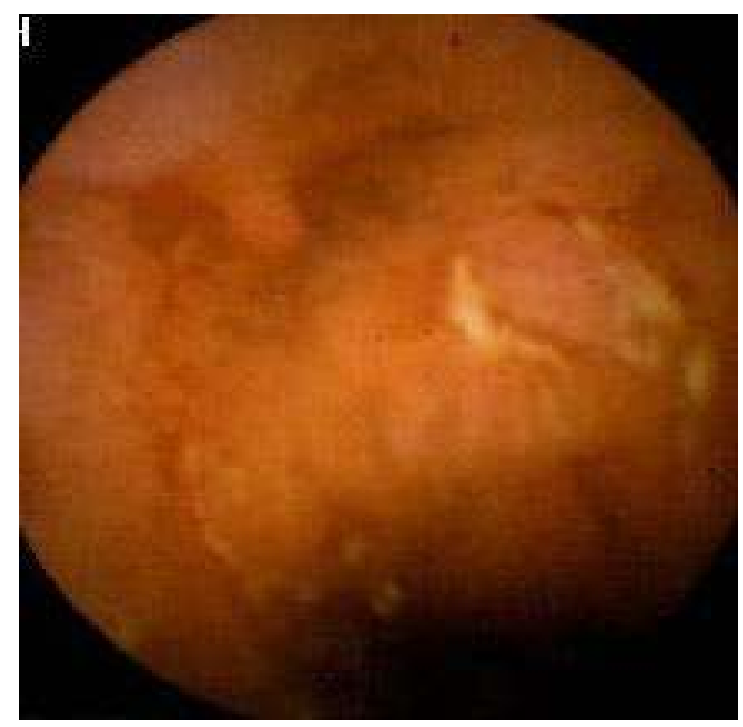

Figure 1 Colonoscopic view showing mucosal ulceration

Colonic biopsy (figure 2) showed moderately altered crypt architecture with extensive mucosal ulceration and suppuration. A large granuloma with pallisading epitheliod cells was seen suggesting intestinal TB or 
Crohn disease. Mantoux test and PCR for tuberculosis of colonic biopsy were negative.

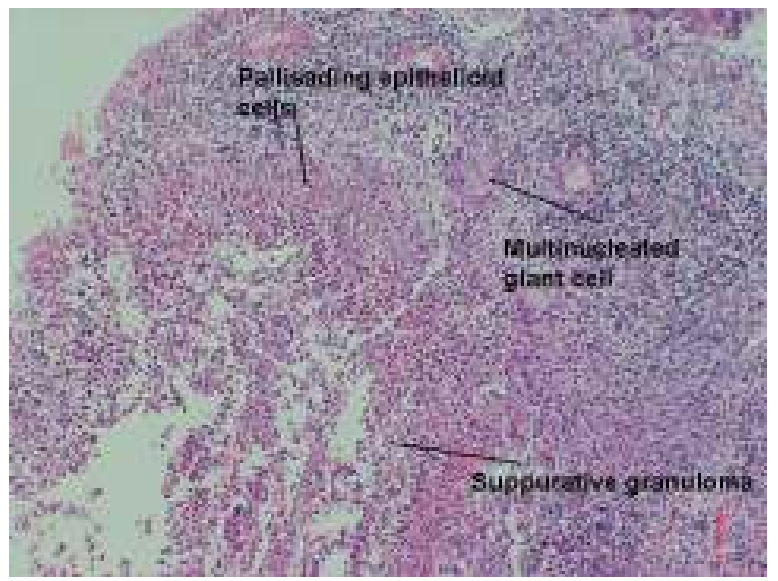

Figure 2 Colonic biopsy showing suppurative granuloma with pallisading epithelioid cells and multinucleated giant cells

A diagnosis of Crohn disease was made at this stage and to study the extent of the involvement of the bowel further imaging was performed. Barium enema (figure 3) showed an oedematous mucosa and a partially obstructed hepatic flexure with skip lesions in the ascending colon demonstrating a characteristic cobblestone appearance.

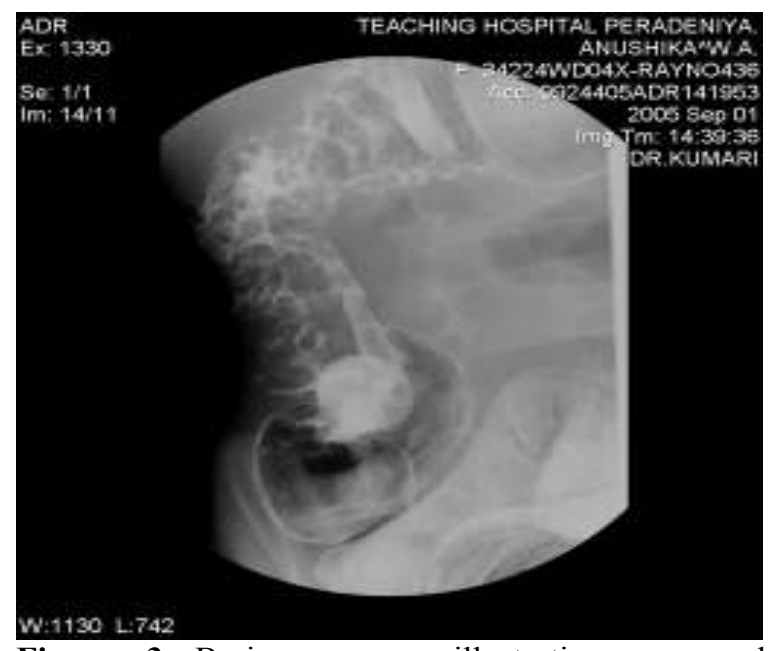

Figure 3 Barium enema illustrating narrowed proximal colon with cobblestone appearance

Barium meal and follow through (figure 4) showed a granular, oedematous mucosa of the stomach and duodenum with cobblestone appearance and a narrowed less distensible ileum. Upper gastrointestinal tract (GIT) endoscopy showed irregularities in the duodenal mucosa. A diagnosis of Crohn's disease with involvement of the stomach, duodenum, ileum, ascending colon, hepatic flexure and transverse colon was made. The child was started on prednisolone $2 \mathrm{mg} / \mathrm{kg}$ and salazopyrin. There was a marked improvement of her clinical condition and haematological parameters including $\mathrm{Hb}, \mathrm{WBC}$, platelet count and ESR with treatment.

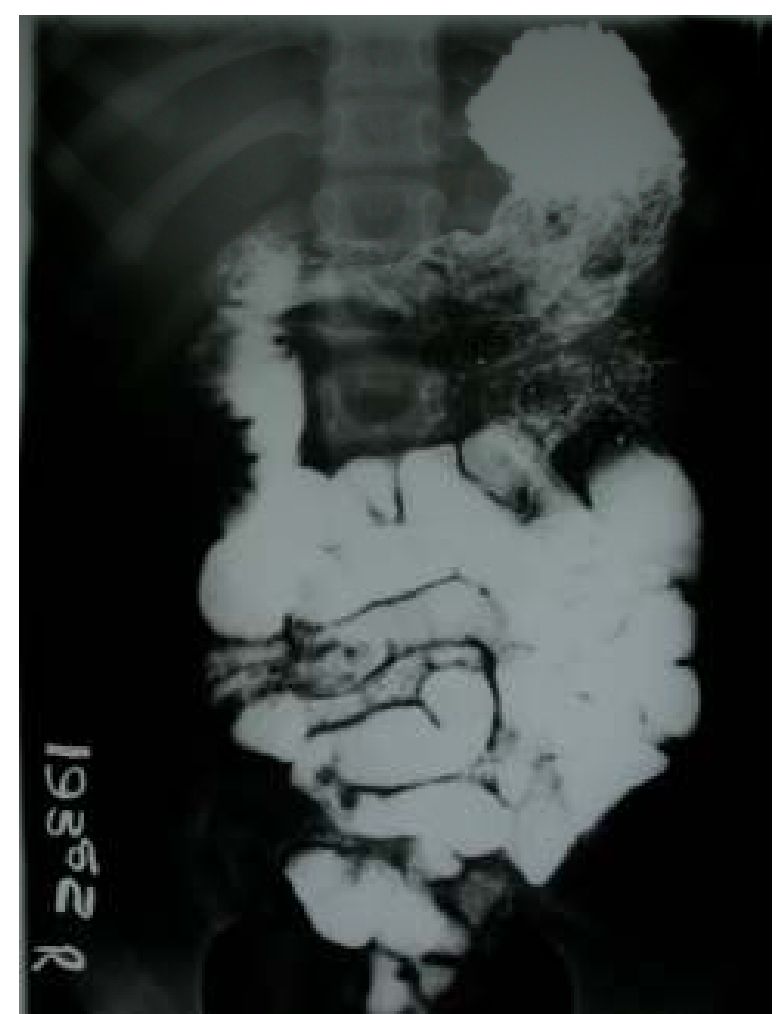

Figure 4 Barium meal showing granular gastric mucosa, duodenal mucosal irregularities with narrowing in the ileac region

\section{Discussion}

Crohn disease, also known as regional ileitis, can present at any age. The aetiology and pathogenesis are unknown despite much research ${ }^{1}$. The genetic linkage studies have identified a number of possible susceptible genes on chromosomes 2, 3, 7 and $16^{1,2,3}$. The HLA correlations are HLA DR 3 and DQ2 that determine the extent of the disease and HLA DR 103 predicts the severity. The role of the environment in the pathogenesis of the disease is not documented ${ }^{2}$.

An abnormality in intestinal immunoregulation may be of primary importance in the pathogenesis of this disease. Most therapies are aimed at interfering with these mediators of inflammation ${ }^{4}$.

In childhood disease, terminal ileitis is common with variable involvement of the colon in $50-70 \% \%^{5}$. There is transmural inflammation with aphthoid and linear ulcers resulting in sinus tract and fistula formation. 
The development of deeper fissures with skip lesions results in the classic cobblestone appearance. Histologically the diagnostic hallmark of the disease is the finding of non-caseating epitheliod granulomata with giant cells ${ }^{5}$.

The insidious onset with non-specific presenting features often result in considerable delay in diagnosis. The importance of growth retardation as the only presenting feature must be emphasized as it may occur in $30 \%$ of children with Crohn disease and may present well before the diagnosis is $\operatorname{made}^{6}$. Other manifestations depend on the site, degree and extent of involvement of the bowel. Perianal disease, when present, is characteristic. Extraintestinal manifestations include aphthous ulcers, peripheral arthritis, iridocyclitis, erythema nodosum, pyoderma gangrenosum, digital clubbing, renal stones, gallstones and sclerosing cholangitis.

Investigations reveal anaemia, often with a component of iron deficiency, elevated ESR and platelet count and a low serum albumin. WBC count may be normal or mildly elevated. Recent studies suggest that measurement of stool calprotectin, a protein secreted into the gut by lymphocytes, is a reliable indicator of intestinal inflammation ${ }^{7}$.

Radiological imaging is helpful to assess the extent of involvement of the bowel. The presence of skip lesions, narrowed lumen, thickening and fissuring with fistula formation of the bowel is highly suggestive of Crohn disease. The value of technetium white cell scanning in the assessment of this disease is debatable ${ }^{8}$. Endoscopy still remains the most important tool for assessing both the upper and lower GIT $^{9}$.

Neither medical nor surgical therapy can cure Crohn disease. Goals of therapy are to relieve symptoms, induce and maintain remission, prevent relapses, correct malnutrition and promote growth.

There is no convincing evidence of a drug that alters the natural history of the disease. Steroids and 5 amino-salicylic compounds are useful in inducing remission but there is very little evidence that continuous therapy will maintain remission. Immunomodulatory drugs (azathioprine and 6mercaptopurine) may be effective in some patients who show a poor response to steroids or are steroid dependant. They may allow reduction or cessation of steroids and help to maintain remission. They are not helpful in the acute stage since their beneficial effects may be delayed by 3-6 months after starting therapy. Infliximab, a chimeric monoclonal antibody to tumour necrosis factor $\alpha$ is a newer agent that is effective in relieving the symptoms and helps to bridge the gap until the immunomodulators take effect ${ }^{10}$.

Nutritional therapy is effective as primary and adjunctive treatment. The enteral nutritional approach is both rapid in onset of response and as effective as other treatments ${ }^{11}$.

It has been said that the best team to treat Crohn disease would be an aggressive physician and a conservative surgeon. Thus, surgical therapy should be reserved only for specific indications. Surgery is indicated for children who fail to respond to medical therapy or its complications. The aim should be to preserve the bowel as much as possible as it can lead to small bowel syndrome ${ }^{12}$. Psychosocial support is important in the adjustment to a difficult problem.

Crohn disease is associated with high morbidity but low mortality. Recurrences may occur without apparent explanation. There is an increased risk of malignancies with long standing disease. Despite these complications most children with Crohn disease lead fully active lives.

\section{Acknowledgements}

We thank Dr. G Buthpitiya, Consultant Surgeon and Senior lecturer in Surgery, Dr. Shanthinie Rosairo, Consultant Radiologist and Senior lecturer and Prof. N.V.I. Ratnatunga, Professor of Pathology of the Faculty of Medicine, University of Peradeniya for their invaluable help.

\section{References}

1. Flocchi C. Inflammatory bowel disease; aetiology and pathogenesis. Gastroenterology 1998; 115:182-205.

2. Tysk C, Lindberg E, Jarnerot G, et.al. Ulcerative colitis and Crohn's disease in selected population of monozygotic and dizygotic twins: a study of heritability and the influence of smoking. Gut 1998; 29: 990-6.

3. Satsangi J, Jewell D P, Bell J I. The genetics of inflammatory bowel disease. Gut 1997; 40: 5724.

4. Griffiths A M. Crohns's disease. Recent adv Paediatr 1992; 10: 145-52. 